\title{
Rethinking the early intubation paradigm of COVID-19: time to change gears?
}

\author{
Philippe Rola ${ }^{1}$, Joshua Farkas ${ }^{2}$, Rory Spiegel ${ }^{3}$, Cameron Kyle-Sidell ${ }^{4}$, \\ Scott Weingart ${ }^{5}$, Laura Duggan ${ }^{6}$, Marco Garrone ${ }^{7}$, Adam Thomas ${ }^{8}$ \\ ${ }^{1}$ Intensive Care Unit, Santa Cabrini Hospital, CIUSSS-CEMTL, Montreal, Canada \\ ${ }^{2}$ Department of Critical Care, University of Vermont, Burlington, VT, USA \\ ${ }^{3}$ Departments of Critical Care and Emergency Medicine, Washington Hospital Center, Georgetown \\ University, Washington, DC, USA \\ ${ }^{4}$ Maimonides Medical Center, Brooklyn, NY, USA \\ ${ }^{5}$ Department of Emergency Medicine, Renaissance School of Medicine, Stony Brook University, Stony Brook, \\ NY, USA \\ ${ }^{6}$ Department of Anesthesiology and Pain Medicine, University of Ottawa, Ottawa, Canada \\ ${ }^{7}$ Emergency Department, Mauriziano Ospedale, Torino, Italy \\ ${ }^{8}$ Adult Critical Care, University of British Columbia, Vancouver, Canada
}

The 21st century's first true pandemic has taken the world by storm in the first months of 2020. Originating in Wuhan, China, the severe acute respiratory syndrome coronavirus 2 (SARS-Cov-2) virus has spread to all corners of the globe in under 4 months and shows little signs of slowing. The medical community has much to thank the Chinese physicians and health care workers-who were the first hit-for organizing a rapid and focused response which included a huge amount of data collection around prevention, diagnosis and management. From this came invaluable information describing this unique illness. ${ }^{1-4}$ While most other forms of acute lung injury present with a somewhat similar clinical pattern of respiratory failure, and if severe enough, are often grouped together in a category we have called acute respiratory distress syndrome or acute respiratory distress syndrome (ARDS).

ARDS tends to be a process of lung inflammation resulting in bilateral alveolar infiltrates, lung unit collapse, ventilation and perfusion mismatch and progressive hypoxia and respiratory distress and failure. The physiological pattern is one of low pulmonary compliance. Great strides were made in the management of these entities by the work of Pelosi et al., ${ }^{5}$ Gattinoni et al., ${ }^{6}$ and Gattinoni et al. ${ }^{7}$ in the 1990's and the advent of the ARDSnet group and the concept of lung

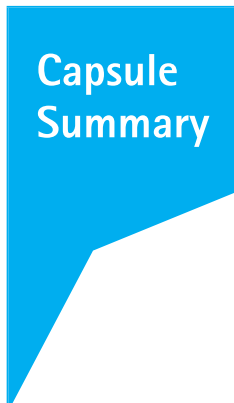

What is already known

It was felt that coronavirus disease 2019 patients needed to be intubated precociously and that is what initial guidelines supported.

What is new in the current study

Current experience suggests that many patients may avoid complications of mechanical ventilation and possibly avoid clinical deterioration by the use of non-invasive ventilatory support.
elSSN: 2383-4625

Received: 5 May 2020

Revised: 11 May 2020

Accepted: 11 May 2020

Correspondence to: Philippe Rola Intensive Care Unit, Santa Cabrini Hospital, CIUSSS-CEMTL, 5655 Rue StZotique, H1T1P7, Montreal, Quebec, Canada

E-mail:philipperola@gmail.com ORCID

https://orcid.org/0000-0002-4425-9212

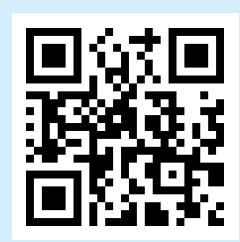

How to cite this article:

Rola P, Farkas J, Spiegel R, Kyle-Sidell C, Weingart S, Duggan L, Garrone M, Thomas A. Rethinking the early intubation paradigm of COVID-19: time to change gears? Clin Exp Emerg Med 2020;7(2):78-80.

This is an Open Access article distributed under the terms of the Creative Commons Attribution Non-Commercial License (https:// creativecommons.org/licenses/by-nc/4.0/). 
protective ventilation, characterized by high positive end expiratory pressure and low tidal volumes. Countless lives were saved using this approach which minimized ventilator-induced lung injury. In the coronavirus disease 2019 (COVID-19) process, however, Gattinoni et al. ${ }^{8}$ recently described a very different pattern with two distinct phenotypes, with only $20 \%$ to $30 \%$ cases evolving to the classic ARDS-like pattern of lung disease, suggesting they need to be managed differently.

The initial message from the Chinese medical teams was to intubate early, somewhere around a 5-6 liter by nasal prong $\mathrm{O}_{2}$ requirement. This seemed to stem from the accurate observation that many of these patients deteriorated precipitously and that they may be more safely intubated at an earlier stage, particularly given the levels of hypoxia encountered during intubation. Additionally, a high work of breathing generating large swings in intrapleural pressure may result in self-inflicted lung injury and worsen the disease process. There is certainly a rationale for early intubation.

However, this also can come at a cost. Mechanical ventilation is inherently associated with a number of well described and accepted complications such as ventilator associated pneumonia, ventilator-induced lung injury, hemodynamic disturbances, as well as all those related to sedation and immobilization.

Interestingly, it appears that SARS-Cov-2 "runs its course" and seems to "turn off" at some point for survivors, who tend to have a two-week course of mechanical ventilation. Hence an early intubation strategy may result in more ventilated days vs a delayed approach.

It appears fair to say that at the very least, there is clinical equipoise as to whether it is best to intubate early before sudden decompensation occurs, or to assess the patient's respiratory strain and whether the clinician feels there is substantial risk of self-inflicted lung injury, and to delay intubation by the use of high flow nasal cannulae or non-invasive ventilation.

Another icon of the ventilatory literature Tobin ${ }^{9}$ noted the need for physiological assessment of the COVID-19 respiratory syndromes and a need to understand that "Respiratory rates of 25 to 35 breaths per minute should not be viewed as ipso facto (knee jerk) justification for intubation."

Additionally, it has become apparent to many clinicians that a significant amount of COVID-19 pneumonia patients present very differently to respiratory failure patients we have been accustomed to see, where the degree of dyspnea correlates relatively well with that of hypoxia. In what has been termed the "happy hypoxic" of SARS-Cov-2, there appears to be an uncoupling of this relationship where the patient is in little or no distress, without tachycardia, yet with profound hypoxia with oxygen satura-
Table 1. Cabrini Respiratory Strain Scale

\begin{tabular}{ll}
\hline Parameter & Point $^{\text {a) }}$ \\
\hline Respiratory rate & \\
$<20$ & 0 \\
$20-30$ & 1 \\
$31-40$ & 2 \\
$>40$ & 4 \\
Retraction/accessory muscle use & \\
None & 0 \\
Mild & 1 \\
Moderate & 2 \\
Severe & 3 \\
Overall state & \\
Relaxed & 0 \\
Uncomfortable & 1 \\
Anxious & 2 \\
Agitated & 3 \\
\hline
\end{tabular}

${ }^{\text {a) } 0-2, ~ l o w ; ~ 3-5, ~ m o d e r a t e ; ~ 6-10, ~ h i g h . ~}$

tions often well below $80 \%$. It is these patients where the wisdom of early intubation is being put into question, with several clinicians opting to tolerate lower saturation goals (variable but generally over 80\%) in the absence of distress.

At Santa Cabrini Hospital in Montreal, a scale of respiratory strain is being used (Cabrini Respiratory Strain Scale) to aid in clinical documentation and in an effort to validate it as a marker of severity and potentially a decision tool for progressing to a higher level of ventilatory support. Data should be forthcoming in the upcoming weeks (Table 1).

Our current ventilatory approach is to delay intubation if it clinically appears safe and feasible. ${ }^{10}$ This requires, ideally, negative pressure rooms and for the patient to be closely monitored in an intensive care unit, given the risk of rapid decompensation, with physicians able to rapidly respond and intubate. We would encourage that other clinicians who are considering this type of management take the time to document cases in an effort to show that, in all likelihood, a proportion of patients can be altogether managed with non-invasive ventilation. Others who fail non-invasive ventilation may still benefit from having potentially a shorter course of mechanical ventilation. This is important data especially in a resource-limited setting as a shorter ventilator course means more ventilators would be available to other patients.

It is important to recognize that this disease is new and that we must find strategies to determine the best course of action as rapidly as possible. The pathophysiology remains unclear, and once intubated, the optimal ventilatory strategy is equally undetermined, with some authors favoring airway pressure release ventilation while others attempt various modes. It is key to keep a keen eye 
on almost daily developments, understanding that guidelines on COVID-19 are, in these early phases, highly fluid constructs. As clinicians we should try to adhere to the principle of individualizing ventilatory therapy to the patient's physiology while avoiding iatrogenic injury.

\section{CONFLICT OF INTEREST}

No potential conflict of interest relevant to this article was reported.

\section{REFERENCES}

1. Wu C, Chen $X$, Cai $Y$, et al. Risk factors associated with acute respiratory distress syndrome and death in patients with coronavirus disease 2019 pneumonia in Wuhan, China. JAMA Intern Med 2020:e200994.

2. Wu Z, McGoogan JM. Characteristics of and important lessons from the coronavirus disease 2019 (COVID-19) outbreak in China: summary of a report of 72314 cases from the Chinese center for disease control and prevention. JAMA 2020; 323:1239-42.

3. Onder G, Rezza G, Brusaferro S. Case-fatality rate and characteristics of patients dying in relation to COVID-19 in Italy. JAMA 2020;323:1775-6.

4. World Health Organization. Report of the WHO-China Joint Mission on Coronavirus Disease 2019 (COVID-19) [Internet].
Geneva: World Health Organization; 2020 [cited 2020 May 4] Available from: https://www.who.int/docs/default-source/ coronaviruse/who-china-joint-mission-on-covid-19-final-report.pdf.

5. Pelosi P, D'Andrea L, Vitale G, Pesenti A, Gattinoni L. Vertical gradient of regional lung inflation in adult respiratory distress syndrome. Am J Respir Crit Care Med 1994;149:8-13.

6. Gattinoni L, Pesenti A, Bombino M, et al. Relationships between lung computed tomographic density, gas exchange, and PEEP in acute respiratory failure. Anesthesiology 1988; 69:824-32.

7. Gattinoni L, Pesenti A, Avalli L, Rossi F, Bombino M. Pressurevolume curve of total respiratory system in acute respiratory failure: computed tomographic scan study. Am Rev Respir Dis 1987;136:730-6.

8. Gattinoni L, Chiumello D, Caironi P, et al. COVID-19 pneumonia: different respiratory treatments for different phenotypes? Intensive Care Med 2020 Apr 14. https://doi.org/10.1007/ s00134-020-06033-2.

9. Tobin MJ. Basing respiratory management of coronavirus on physiological principles. Am J Respir Crit Care Med 2020;201: 1319-20.

10. Weingart S. ThinkingCC COVID respiratory management webinar [Internet]. EMCrit RACC Blog; 2020 Apr 12 [cited 2020 May 4]. Available from: https://emcrit.org/emcrit/covid-respiratory-management/. 